\title{
ON THE CAUCHY PROBLEM FOR THE 1+2 COMPLEX GINZBURG-LANDAU EQUATION
}

\author{
CHARLES BU ${ }^{1}$
}

(Received 31 August 1992; revised 1 May 1993)

\begin{abstract}
We present analytical methods to investigate the Cauchy problem for the complex GinzburgLandau equation $u_{t}=(\nu+i \alpha) \Delta u-(\kappa+i \beta)|u|^{2 q} u+\gamma u$ in 2 spatial dimensions (here all parameters are real). We first obtain the local existence for $\nu>0, \kappa \geq 0$. Global existence is established in the critical case $q=1$. In addition, we prove the global existence when $q=2$ if (1) $|\beta| \leq \frac{\sqrt{5}}{2} \kappa$ or (2) $\alpha \beta>0$.
\end{abstract}

\section{Introduction}

The complex Ginzburg-Landau equation (GL)

$$
u_{t}=(\nu+i \alpha) \Delta u-(\kappa+i \beta)|u|^{2 q} u+\gamma u
$$

with a $2 q+1$ order nonlinearity in $D$ spatial dimensions in the domain $\Omega \subset R^{D}$ was originally derived by Newell and Whitehead $[15,17]$ and studied by Hocking, Stewartson and Stuart $[13,22]$ to describe the amplitude evolution of instable waves in fluid dynamics. The critical case $q D=2$ is the key to understanding (1.1) (cf. [2]). It is important to realize that $\nu, \kappa \geq 0$ is often necessary to establish a solution which is physically meaningful. If $\nu=\kappa=\gamma=0$ one has the nonlinear Schrödinger equation (NLS limit). It is well-known that a necessary condition for the blowup of solutions to the NLS limit with certain nontrivial initial data is $q D \geq 2$. (For details on the Cauchy problem of the NLS limit in D spatial dimensions, see H. Brezis and T. Gallouet [3], M. Tsutsumi [25], W. Strauss [23], Ablowitz and Segur [1].) For $q=D=1$, the Cauchy problem for the GL equation with $x \in \Omega=[0, L], u(x, 0) \in H_{0}^{1}(\Omega)$ is wellposed as can be seen by using classical techniques of nonlinear parabolic equations (cf. $[12,16])$. One could also study the GL equation as a perturbed NLS limit to investigate the phenomena associated with singular perturbation of the NLS [18]. For

'Department of Mathematics, Wellesley College, Wellesley, Massachusetts 02181, U.S.A.

(C) Australian Mathematical Society, 1994, Serial-fee code 0334-2700/94 
$\Omega=[0, \infty)$ it is possible to impose initial and boundary conditions such that $u(x, 0)$ and $u(0, t)$ are given and study the half-line GL equation for $x, t \geq 0$. This problem is interesting from a certain experimental point of view. For the NLS limit, such a problem is called a forced integrable system (see Kaup [14]). It can be solved via inverse scattering (e.g. Carroll [7], Fokas [11]) and semigroup theory ([8], [4]). For the half-line GL equation, a unique global solution is established if $|\beta| \leq \sqrt{3} \kappa$ or $\alpha \beta>0$ (cf. [5]). For $D=2$, numerous results are available in the literature, but nearly all of them require that $\Omega$ be a finite domain, for example, $\Omega=[0,1]^{D}$. The recent joint paper by Bartuccelli, Constantin, Doering, Gibbon and Gisselfalt [2] gives many interesting estimates in their study of the possibility of soft and hard turbulence in the GL equation with $q=1$ and $\Omega=[0,1]^{2}$. For the initial (or boundary) value problems in an unbounded domain, much is unknown.

This paper is devoted to $q=1$ (the critical case) and $q=2$ (the super-critical case) with $D=2$, and $\Omega=R^{2}$ throughout. When the boundary is pushed to infinity, we would like to see under which restriction on the coefficients the system tries to blow up, only apparently being pulled back by dissipation. We note that the complex Ginzburg-Landau equation (1.1) could be derived as a wave envelope or amplitude equation governing wave-packet solutions, for example, in the study of the Taylor-Couette flow, Benard convection and plane Poiseuille flow. It is well-known that there is a significance difference in behavior of the hard and soft turbulence in the system when we move from $D=1$ to $D=2$. This change is caused by the background role of the NLS equation, which is the dissipationless limit of the complex Ginzburg-Landau equation. For $D=1$, the NLS is integrable and has infinitely many conserved quantities, but when $D \geq 2$ solutions of the NLS might blow up under certain conditions. We note that it is generally more difficult to treat the unbounded domain problem than the bounded domain problem. For example, to disprove the global existence, one would like to show the blow-up of the solution. However, when the domain is unbounded, it is much harder to do that.

The main results are summarized as follows. We first in Section 2 establish a unique local classical solution to the Cauchy problem of the GL equation (1.1) with initial data $u(x, y, 0)=u_{0}(x, y) \in H^{3}(\Omega)$. In Sections 3 and 4 we prove the global existence theorems. The first global existence theorem is shown when $q=1$. The second and third global existence theorems are based on the criterion $|\beta| \leq \frac{\sqrt{5}}{2} \kappa$ or $\alpha \beta>0$ when $q=2$. The physical significance of these criteria is also discussed.

\section{Existence of the local solution}

The Cauchy problem for the Ginzburg-Landau equation in 2 spatial dimensions is posed as follows ( $\kappa>0, \nu \geq 0, \alpha, \beta, \gamma$ real): 


$$
\begin{aligned}
u_{t} & =(\nu+i \alpha) \Delta u-(\kappa+i \beta)|u|^{2 q} u+\gamma u, \\
u(x, y, 0) & =u_{0}(x, y), \quad(x, y) \in \Omega=R^{2}, \quad t \geq 0 .
\end{aligned}
$$

Here $u_{0}(x, y)$ is a smooth complex function. We shall, throughout this paper, assume that

$$
\begin{aligned}
\|u\|_{p} & =\left[\int_{-\infty}^{\infty} \int_{-\infty}^{\infty}|u|^{p} d x d y\right]^{\frac{1}{p}}, \\
\|\nabla u\|_{2} & =\left[\int_{-\infty}^{\infty} \int_{-\infty}^{\infty}|\nabla u|^{2} d x d y\right]^{\frac{1}{2}}, \\
\|\Delta u\|_{2} & =\left[\int_{-\infty}^{\infty} \int_{-\infty}^{\infty}|\Delta u|^{2} d x d y\right]^{\frac{1}{2}} .
\end{aligned}
$$

We first write down the Gagliardo-Nirenberg estimates [19] which relate the $L^{p}$ norm of the $j$ th derivative of $u$ to other norms and their derivatives:

$$
\left\|\nabla^{j} u\right\|_{p} \leq c\left\|\nabla^{m} u\right\|_{r}^{a}\|u\|_{q}^{1-a},
$$

where

$$
\frac{1}{p}=\frac{j}{D}+a\left(\frac{1}{r}-\frac{m}{D}\right)+\frac{1-a}{q}
$$

with $1 \leq q, r \leq \infty$. Two further restrictions are $0 \leq j<m$ and $j / m \leq a<1$. We will assume $D=2$ throughout this paper. Thus by setting $p=6, j=0, m=1$, $r=q=2$ in (2.5) one has

$$
\|u\|_{6} \leq c\|\nabla u\|_{2}^{\frac{2}{3}}\|u\|_{2}^{\frac{1}{3}} .
$$

By setting $p=\infty, j=0, m=3, r=q=2$ in (2.5) one has

$$
\|u\|_{\infty} \leq c_{1}\left\|\nabla^{3} u\right\|_{2}^{\frac{1}{3}}\|u\|_{2}^{\frac{2}{3}},
$$

and by setting $p=2, j=1, m=3, r=q=2$ one obtains

$$
\|\nabla u\|_{2} \leq c_{2}\left\|\nabla^{3} u\right\|_{2}^{\frac{1}{3}}\|u\|_{2}^{\frac{2}{3}} \text {. }
$$

Also by setting $p=\infty, j=0, m=r=q=2$ one obtains

$$
\|u\|_{\infty} \leq c_{3}\left\|\nabla^{2} u\right\|_{2}^{\frac{1}{2}}\|u\|_{2}^{\frac{1}{2}} .
$$

Finally, by setting $p=4, j=0, m=r=q=2$ one has

$$
\|u\|_{6} \leq c_{4}\left\|\nabla^{2} u\right\|_{2}^{\frac{1}{3}}\|u\|_{2}^{\frac{2}{3}} .
$$

The above five estimates (2.7), (2.8), (2.9) (2.10) and (2.11) will be used in Sections 3 and 4 to prove global existence. 
LeMMA 2.1. Let $A u=(v+i \alpha) \Delta u, D(A)=H^{2}\left(R^{2}\right)$. Then the operator $A$ is the infinitesimal generator of a continuous semigroup of contractors $N(t)=\exp$ At for $t \geq 0$.

Proof. Let $H=L^{2}\left(R^{2}\right), V=H^{1}\left(R^{2}\right)$, then $D(A)$ and $V$ are dense in $H$. To show that the resolvent set of $A$ contains $R^{+}$, let $v \in V, \lambda>0$. Consider

$$
\begin{aligned}
((\lambda-A) v, v) & =\int_{-\infty}^{\infty} \int_{-\infty}^{\infty}(\lambda v-(\nu+i \beta) \Delta v) \bar{v} d x d y \\
& =\lambda\|v\|_{2}^{2}+(\nu+i \beta)\|\nabla v\|_{2}^{2} .
\end{aligned}
$$

Take the real part of (2.12). One has

$$
|((\lambda-A) v, v)| \geq \lambda\|v\|_{2}^{2}+v\|\nabla v\|_{2}^{2} \geq c_{0}\|v\|_{V}^{2} .
$$

and by the Lax-Milgram Theorem [6], the operator $\lambda-A$ maps $D(A) 1-1$ onto $H$. Now let $v \in D(A)$. From (2.13) one has the inequality $\lambda\|v\|_{2} \leq\|(\lambda-A) v\|_{2}$. Thus $\left\|(\lambda-A)^{-1}\right\| \leq 1 / \lambda$. By Hille-Yosida Theorem [20, Section 1.3.1]), the unbounded and linear operator $A$ is the infinitesimal generator of a countinuous semigroup of contractors $N(t)=\exp A t$ for $t \geq 0$.

Now one could convert (2.1) to an integral equation

$$
\begin{aligned}
u & =N(t) u_{0}+\int_{0}^{t} N(t-s)\left[-(\kappa+i \beta)|u|^{2} u+\gamma u\right] d s \\
& =N(t) u_{0}+\int_{0}^{t} N(t-s) G(s) d s .
\end{aligned}
$$

Since $G(t)=-(\kappa+i \beta)|u|^{2} u+\gamma u$ is locally Lipschitz in $u$ under the norm of $D(A)$ uniformly on $[0, T]$ for any $T>0$, by [20, Theorem 6.1 .7$]$ one obtains the following local existence theorem:

THEOREM 2.2. For $u_{0}(x, y) \in D(A)$, there exists a unique solution $u$ to (2.1) such that $u \in C^{0}\left(\left[0, T_{M}\right), D(A)\right) \cap C^{1}\left(\left[0, T_{M}\right), L^{2}\right)$ with either $\lim \|u\|_{D(A)}=\infty$ as $t \rightarrow T_{M}^{-}$ or $T_{M}=\infty$.

REMARK 2.3. By the regularity theory [21], if $u_{0}(x, y) \in H^{3}\left(R^{2}\right)$ then the solution (obtained by Theorem 2.2) $u \in C^{0}\left(\left[0, T_{M}\right), H^{3}\left(R^{2}\right)\right) \cap C^{1}\left(\left[0, T_{M}\right), H^{1}\left(R^{2}\right)\right.$ ).

In next three sections we will prove the global existence in the following situations: (1) $q=1$; (2) $q=2$ and $|\beta| \leq \frac{\sqrt{5}}{\kappa}$; (3) $q=2$ and $\alpha \beta>0$. The main tools are the Gagliardo-Nirenberg estimates and various estimates demonstrated in Sections 3 and 4. Some of these estimates require that $u \in H^{3}$. 


\section{Global existence theorem (I) in the case $q=1$}

The purpose of this section is to prove the global existence of the Cauchy problem for the GL equation in the critical case $q=1$.

THEOREM 3.1. Suppose $q=1$ and $u_{0}(x, y) \in H^{3}\left(R^{2}\right)$. Then $T_{M}=\infty$.

PROOF. Three steps are needed in the proof, that is, to show that on any given interval $[0, T], 1)\|u\|_{2}$ is bounded; 2) $\|\nabla u\|_{2}$ is bounded and 3) $\left\|\nabla^{2} u\right\|_{2}$ is bounded and hence $\|u\|_{D(A)}$ is bounded.

STEP 1. The following could be directly verified (noting $\kappa>0, v \geq 0$ ):

$$
\partial_{t}\|u\|_{2}^{2}=-2 \kappa\|u\|_{4}^{4}+2 \gamma\|u\|_{2}^{2}-2 v\|\nabla u\|_{2}^{2} \leq 2 \gamma\|u\|_{2}^{2} .
$$

By Gronwall's lemma, for $t \in[0, T]$,

$$
\|u\|_{2}^{2} \leq\left\|u_{0}\right\|_{2}^{2} \exp \{2|\gamma| t\} \leq\left\|u_{0}\right\|_{2}^{2} \exp \{2|\gamma| T\}=M_{0}^{2} .
$$

STEP 2. Calculate

$$
\begin{aligned}
\partial_{t}\left\|u_{x}\right\|_{2}^{2} & =2 \operatorname{Re} \int_{-\infty}^{\infty} \int_{-\infty}^{\infty} u_{t x} \bar{u}_{x} d x d y \\
& =-2 \operatorname{Re} \int_{-\infty}^{\infty} \int_{-\infty}^{\infty} u_{t} \bar{u}_{x x} d x d y \\
& =-2 \operatorname{Re} \int_{-\infty}^{\infty} \int_{-\infty}^{\infty}\left[(v+i \alpha)\left(u_{x x}+u_{y y}\right) \bar{u}_{x x}\right. \\
& \left.\quad-(\kappa+i \beta)|u|^{2} u \bar{u}_{x x}+\gamma u \bar{u}_{x x}\right] d x d y \\
& \leq-2 v\left\|u_{x x}\right\|_{2}^{2}-2 v\left\|u_{x y}\right\|_{2}^{2}+c^{\prime}\left\|+6^{6}+v\right\| u_{x x}\left\|_{2}^{2}+2 \gamma\right\| u_{x} \|_{2}^{2} .
\end{aligned}
$$

Therefore

$$
\partial_{t}\|\nabla u\|_{2}^{2} \leq-v\|\Delta u\|_{2}^{2}+2 c^{\prime}\|u\|_{6}^{6}+2 \gamma\|\nabla u\|_{2}^{2} .
$$

Since $\|u\|_{6}^{6} \leq c^{6}\|\Delta u\|_{2}^{4}\|u\|_{2}^{2}$ from (2.7), we work on (3.4):

$$
\partial_{t}\|\nabla u\|_{2}^{2} \leq-v\|\Delta u\|_{2}^{2}+2 c^{\prime} c^{6}\|\nabla u\|_{2}^{4} u t^{2}+2 \gamma\|\nabla u\|_{2}^{2} .
$$

Let $g(t)=1+\|\nabla u\|_{2}^{2}$. It is evident from (3.1) and (3.2) that $\|u\|_{2} \leq M_{0}$ and

$$
\begin{aligned}
\int_{0}^{t}\|\nabla u\|_{2}^{2} d \tau & \leq\|u\|_{2}^{2}+\left\|u_{0}\right\|_{2}^{2}+2|\gamma| \int_{0}^{t}\|u\|_{2}^{2} d \tau \\
& \leq 2 M_{0}^{2}+2|\gamma| M_{0}^{2} t .
\end{aligned}
$$


By (3.5) one obtains

$$
g^{\prime}(t) \leq-v\|\Delta u\|_{2}^{2}+c_{0} g^{2}(t)+h(t),
$$

where $h(t)$ is a continuous function on $[0, \infty)$. Ignoring the $-v\|\Delta u\|_{2}^{2}$ norm, divide both sides of (3.7) by $g(t)$ and integrate to get

$$
g(t) \leq g(0) \exp \left(\int_{0}^{t}\left(c_{0} g(\tau)+h(\tau)\right) d \tau\right.
$$

One concludes from (3.6) and (3.8) that $\|\nabla u\|_{2}^{2}$ is bounded on any $[0, T]$. Further, we can see that $\int_{0}^{t}\|\Delta u\|_{2}^{2} d \tau$ is also bounded for any $t>0$.

STEP 3. Consider

$$
\begin{aligned}
\partial_{t} & \int_{-\infty}^{\infty} \int_{-\infty}^{\infty}\left|u_{x x}\right|^{2} d x d y \\
& =-\partial_{t} \int_{-\infty}^{\infty} \int_{-\infty}^{\infty} u_{x} \bar{u}_{x x x} d x d y \\
& =-2 \operatorname{Re} \int_{-\infty}^{\infty} \int_{-\infty}^{\infty} u_{x t} \bar{u}_{x x x} d x d y \\
& =-2 \operatorname{Re} \int_{-\infty}^{\infty} \int_{-\infty}^{\infty}\left((v+i \alpha) \Delta u-(\kappa+i \beta)|u|^{2} u+\gamma u\right)_{x} \bar{u}_{x x x} d x d y \\
& \leq-2 v\left\|u_{x x x}\right\|_{2}^{2}+2 \operatorname{Re} \int_{-\infty}^{\infty} \int_{-\infty}^{\infty}\left((\kappa+i \beta)\left(2|u|^{2} u_{x}+u^{2} \bar{u}_{x}\right)-\gamma u_{x}\right) \bar{u}_{x x x} d x d y \\
& \leq-2 v\left\|u_{x x x}\right\|_{2}^{2}+2 \int_{-\infty}^{\infty} \int_{-\infty}^{\infty}\left(3|\kappa+i \beta||u|^{2}\left|u_{x}\right|+\left|\gamma u_{x}\right|\right)\left|u_{x x x}\right| d x d y \\
& \leq-2 v\left\|u_{x x x}\right\|_{2}^{2}+\int_{-\infty}^{\infty} \int_{-\infty}^{\infty}\left(\frac{(3|\kappa+i \beta|)^{2}}{v}|u|^{4}\left|u_{x}\right|^{2}+\frac{\left|\gamma u_{x}\right|^{2}}{v}\right) d x d y+v\left\|u_{x x x}\right\|_{2}^{2} \\
& \leq \int_{-\infty}^{\infty} \int_{-\infty}^{\infty}\left(\frac{(3|\kappa+i \beta|)^{2}}{v}|u|^{4}\left|u_{x}\right|^{2}+\frac{\left|\gamma u_{x}\right|^{2}}{v}\right) d x d y
\end{aligned}
$$

and use (2.10) to estimate $|u(x, y, t)|$ in (3.9):

$$
\begin{aligned}
\partial_{t} \int_{-\infty}^{\infty} \int_{-\infty}^{\infty}\left|u_{x x}\right|^{2} d x d y \\
\quad \leq \int_{-\infty}^{\infty} \int_{-\infty}^{\infty}\left(\frac{(3|\kappa+i \beta|)^{2}}{v}\|u\|_{\infty}^{4}\left|u_{x}\right|^{2}+\frac{\left|\gamma u_{x}\right|^{2}}{v}\right) d x d y \\
\quad \leq \int_{-\infty}^{\infty} \int_{-\infty}^{\infty} \frac{(3|\kappa+i \beta|)^{2}}{v}\left(c_{3}\left\|\nabla^{2} u\right\|_{2}^{\frac{1}{2}}\|u\|_{2}^{\frac{1}{2}}\right)^{4}\left|u_{x}\right|^{2} d x d y+\frac{|\gamma|^{2}}{v}\left\|u_{x}\right\|^{2} \\
\quad=\tilde{c}\left\|\nabla^{2} u\right\|_{2}^{2}\|u\|_{2}^{2}\left\|u_{x}\right\|_{2}^{2}+c^{\prime}\left\|u_{x}\right\|_{2}^{2} .
\end{aligned}
$$


Therefore by (3.2), (3.8) and (3.10)

$$
\begin{aligned}
\partial_{t}\left(\left\|u_{x x}\right\|_{2}^{2}+\left\|u_{y y}\right\|_{2}^{2}\right) & \leq \tilde{c}\left\|\nabla^{2} u\right\|_{2}^{2}\|u\|_{2}^{2}\|\nabla u\|_{2}^{2}+c^{\prime}\|\nabla u\|_{2}^{2} \\
& \leq \tilde{c}\left\|\nabla^{2} u\right\|_{2}^{2} M_{0}^{2} M_{1}^{2}+c^{\prime} M_{1}^{2} .
\end{aligned}
$$

Since the $\|\Delta u\|_{2}$-norm and $\left\|\nabla^{2} u\right\|_{2}$-norm are equivalent, by applying Gronwall's inequality to (3.11) one obtains

$$
\left\|\nabla^{2} u\right\|_{2}^{2} \leq \hat{c} \exp \{\bar{c} t\} \leq \hat{c} \exp \{\bar{c} T\}=M_{2}^{2}
$$

for $0 \leq t \leq T$. From (3.2), (3.7) and (3.11) we conclude that $\|u\|_{D(A)}$ is bounded on $[0, T]$ for any $T>0$. By Theorem $2.2, T_{M}=\infty$.

\section{Global existence theorems (II) and (III) in the case $q=2$}

This purpose of this section is to prove the global existence of the Cauchy problem for the GL equation if $|\beta| \leq \frac{\sqrt{5}}{2}$ or $\alpha \beta>0$ when $q=2$.

THEOREM 4.1. Suppose $u_{0}(x, y) \in H^{3}\left(R^{2}\right)$ and $|\beta| \leq \frac{\sqrt{5}}{2}$. Then $T_{M}=\infty$.

ProOF. We will show that on any given interval (1) $\|u\|_{2}$ is bounded, (2) $\|\nabla u\|_{2}$ is bounded, (3) $\|\Delta u\|_{2}$ is bounded and hence $\|u\|_{D(A)}$ is bounded.

One verifies the following directly:

$$
\partial_{t}\|u\|_{2}^{2}=-2 \kappa\|u\|_{6}^{6}+2 \gamma\|u\|_{2}^{2}-2 v\|\nabla u\|_{2}^{2} \leq 2 \gamma\|u\|_{2}^{2} .
$$

By Gronwall's lemma, for $t \in[0, T]$,

$$
\|u\|_{2}^{2} \leq\left\|u_{0}\right\|_{2}^{2} \exp (2|\gamma| t) \leq\left\|u_{0}\right\|_{0}^{0} \exp (2|\gamma| T)=M_{0}^{2} .
$$

As a matter of fact, we also see that $\int_{0}^{t}\|\nabla u\|_{2}^{2} d \tau$ is bounded for any $t>0$ from (4.1) and (4.2) since $v>0$. Now we turn to

$$
\begin{aligned}
\partial_{t}\left\|u_{x}\right\|_{2}^{2} & =2 \operatorname{Re} \int_{-\infty}^{\infty} \int_{-\infty}^{\infty} u_{t x} \bar{u}_{x} d x d y=-2 \operatorname{Re} \int_{-\infty}^{\infty} \int_{-\infty}^{\infty} u_{t} \bar{u}_{x x} d x d y \\
& =-2 \operatorname{Re} \int_{-\infty}^{\infty} \int_{-\infty}^{\infty}\left[(v+i \alpha)\left(u_{x x}+u_{y y}\right) \bar{u}_{x x}-(\kappa+i \beta)|u|^{4} u \bar{u}_{x x}\right] d x d y \\
\leq & -2 v\left\|u_{x x}\right\|_{2}^{2}-2 v\left\|u_{x y}\right\|_{2}^{2}+2 \operatorname{Re}(\kappa+i \beta) \int_{-\infty}^{\infty} \int_{-\infty}^{\infty} u^{3} \bar{u}^{2} \bar{u}_{x x} d x d y \\
& +2 \gamma \int_{-\infty}^{\infty} \int_{-\infty}^{\infty}\left|u_{x}\right|^{2} d x d y .
\end{aligned}
$$


Let $u \bar{u}_{x}=A+i B$ and consider

$$
\begin{aligned}
2 \operatorname{Re}(\kappa & +i \beta) \int_{-\infty}^{\infty} \int_{-\infty}^{\infty} u^{3} \bar{u}^{2} \bar{u}_{x x} d x d y \\
& =-2 \operatorname{Re}(\kappa+i \beta) \int_{-\infty}^{\infty} \int_{-\infty}^{\infty}|u|^{2}\left(3|u|^{2}\left|u_{x}\right|^{2}+2 u^{2} \bar{u}_{x}^{2}\right) d x d y \\
& =-2 \int_{-\infty}^{\infty} \int_{-\infty}^{\infty}|u|^{2}\left(3 \kappa|u|^{2}\left|u_{x}\right|^{2}+2 \kappa \operatorname{Re} u^{2} \bar{u}_{x}^{2}-2 \beta \operatorname{Im} u^{2} \bar{u}_{x}^{2}\right) d x d y \\
& =-2 \int_{-\infty}^{\infty} \int_{-\infty}^{\infty}|u|^{2}\left(3 \kappa\left(A^{2}+B^{2}\right)+2 \kappa\left(A^{2}-B^{2}\right)-4 \beta A B\right) d x d y \\
& =-2 \int_{-\infty}^{\infty} \int_{-\infty}^{\infty}|u|^{2}\left(5 \kappa A^{2}+\kappa B^{2}-4 \beta A B\right) d x d y .
\end{aligned}
$$

Since $|\beta| \leq \frac{\sqrt{5}}{2} \kappa$, one observes that the above quantity is negative definite. Thus (4.3) becomes

$$
\begin{aligned}
\partial_{t}\left\|u_{x}\right\|_{2}^{2} & \leq-2 v\left\|u_{x x}\right\|_{2}^{2}-2 v\left\|u_{x y}\right\|_{2}^{2}+2 \gamma \int_{-\infty}^{\infty} \int_{-\infty}^{\infty}\left|u_{x}\right|^{2} d x d y \\
& \leq 2 \gamma \int_{-\infty}^{\infty} \int_{-\infty}^{\infty}\left|u_{x}\right|^{2} d x d y .
\end{aligned}
$$

One concludes from (4.5) that

$$
\partial_{\ell}\|\nabla u\|_{2}^{2} \leq 2 \gamma\|\nabla u\|_{2}^{2}
$$

Apply Gronwall's inequality to (4.6) to obtain

$$
\|\nabla u\|_{2}^{2} \leq\left\|\nabla u_{0}\right\|_{2}^{2} \exp \{2|\gamma| t\} \leq\left\|\nabla u_{0}\right\|_{2}^{2} \exp \{2|\gamma| T\} \leq M_{1}^{2} .
$$

Write

$$
\begin{aligned}
& \partial_{t} \int_{-\infty}^{\infty} \int_{-\infty}^{\infty}\left|u_{x x}\right|^{2} d x d y \\
& \quad=-\partial_{t} \int_{-\infty}^{\infty} \int_{-\infty}^{\infty} u_{x} \bar{u}_{x x x} d x d y \\
& \quad=-2 \operatorname{Re} \int_{-\infty}^{\infty} \int_{-\infty}^{\infty} u_{x t} \bar{u}_{x x x} d x d y \\
& \quad=-2 \operatorname{Re} \int_{-\infty}^{\infty} \int_{-\infty}^{\infty}\left((v+i \alpha) \Delta u-(\kappa+i \beta)|u|^{4} u+\gamma u\right)_{x} \bar{u}_{x x x} d x d y \\
& \quad \leq-2 v\left\|u_{x x x}\right\|_{2}^{2}+2 \operatorname{Re} \int_{-\infty}^{\infty} \int_{-\infty}^{\infty}\left((\kappa+i \beta)\left(3|u|^{4} u_{x}+2|u|^{2} u^{2} \bar{u}_{x}\right)-\gamma u_{x}\right) \bar{u}_{x x x} d x d y
\end{aligned}
$$




$$
\begin{aligned}
& \leq-2 v\left\|u_{x x x}\right\|_{2}^{2}+2 \int_{-\infty}^{\infty} \int_{-\infty}^{\infty}\left(5|\kappa+i \beta||u|^{4}\left|u_{x}\right|+\left|\gamma u_{x}\right|\right)\left|u_{x x x}\right| d x d y \\
& \leq-2 v\left\|u_{x x x}\right\|_{2}^{2}+\int_{-\infty}^{\infty} \int_{-\infty}^{\infty}\left(\frac{(5|\kappa+i \beta|)^{2}}{v}|u|^{8}\left|u_{x}\right|^{2}+\frac{\left|\gamma u_{x}\right|^{2}}{v}\right) d x d y+v\left\|u_{x x x}\right\|_{2}^{2} \\
& \leq \int_{-\infty}^{\infty} \int_{-\infty}^{\infty}\left(\frac{(5|\kappa+i \beta|)^{2}}{v}|u|^{8}\left|u_{x}\right|^{2}+\frac{\left|\gamma u_{x}\right|^{2}}{v}\right) d x d y
\end{aligned}
$$

and use (2.10) to estimate $|u(x, y, t)|$ in (4.8):

$$
\begin{aligned}
\partial_{t} \int_{-\infty}^{\infty} & \int_{-\infty}^{\infty}\left|u_{x x}\right|^{2} d x d y \\
& \leq \int_{-\infty}^{\infty} \int_{-\infty}^{\infty}\left(\frac{(5|\kappa+i \beta|)^{2}}{v}\|u\|_{\infty}^{8}\left|u_{x}\right|^{2}+\frac{\left|\gamma u_{x}\right|^{2}}{v}\right) d x d y \\
& \leq \int_{-\infty}^{\infty} \int_{-\infty}^{\infty} \frac{(5|\kappa+i \beta|)^{2}}{v}\left(c_{3}\left\|\nabla^{2} u\right\|_{2}^{\frac{1}{2}}\|u\|_{2}^{\frac{1}{2}}\right)^{8}\left|u_{x}\right|^{2} d x d y+\frac{|\gamma|^{2}}{v}\left\|u_{x}\right\|^{2} \\
& =\tilde{c}\left\|\nabla^{2} u\right\|_{2}^{4}\|u\|_{2}^{4}\left\|u_{x}\right\|_{2}^{2}+c^{\prime}\left\|u_{x}\right\|_{2}^{2}
\end{aligned}
$$

Therefore by (4.2), (4.7) and (4.9)

$$
\begin{aligned}
\partial_{t}\left(\left\|u_{x x}\right\|_{2}^{2}+\left\|u_{y y}\right\|_{2}^{2}\right) & \leq \tilde{c}\left\|\nabla^{2} u\right\|_{2}^{4}\|u\|_{2}^{4}\|\nabla u\|_{2}^{2}+c^{\prime}\|\nabla u\|_{2}^{2} \\
& \leq \tilde{c}\left\|\nabla^{2} u\right\|_{2}^{4} M_{0}^{4} M_{1}^{2}+c^{\prime} M_{1}^{2}
\end{aligned}
$$

Since the $\|\Delta u\|_{2}$-norm and $\left\|\nabla^{2} u\right\|_{2}$-norm are equivalent, by setting up $g(t)=1+$ $\left\|\nabla^{2} u\right\|_{2}^{2}+1$ in (4.10), dividing both sides by $g(t)$ and integrating from 0 to $t$, one obtains

$$
\left\|\nabla^{2} u\right\|_{2}^{2} \leq g(t) \leq g(0) \exp \left(c_{0} t+\int_{0}^{t} g(\tau) d \tau\right) .
$$

From (4.5) it is clear that $\int_{0}^{t} g(\tau) d \tau$ is bounded for any $t>0$ and hence $\left\|\nabla^{2} u\right\|_{2}^{2}$ is bounded on any $[0, T]$. We therefore conclude that $\|u\|_{D(A)}$ is bounded on $[0, T]$ for any $T>0$ and the solution is global.

REMARK 4.2. The following rescaled Ginzburg-Landau equation

$$
A_{t}=R A+(1+i v) \Delta u-(1+i \mu)|A|^{4} A
$$

has been studied extensively where $v=\epsilon a, \mu=\epsilon b$. It leads to a perturbation analysis on a complex Duffing equation. The criterion $|\beta| \leq \frac{\sqrt{5}}{\kappa}$ is met since $\beta=\epsilon b, \kappa=1$ and $\epsilon \ll 1$. Thus the Cauchy problem for $(*)$ has a unique global solution provided that $u_{0}(x, y) \in H^{3}$.

Our next global existence theorem is based on the criterion $\alpha \beta>0$. 
THEOREM 4.3. If $q=2, u_{0}(x, y) \in H^{3}\left(R^{2}\right)$ and $\alpha \beta>0$ then $T_{M}=\infty$.

PROOF. Without loss of generality, let us assume that $\alpha>0, \beta>0$. Again we don't have to worry about Step 1 since $\nu>0, \kappa \geq 0$. To proceed with Step 2, differentiate the second Lyapunov functional $F_{2}$ defined in [2] with respect to $t$ :

$$
\begin{aligned}
& \partial_{t} F_{2}(t)= \partial_{t}\left(\|\nabla u\|_{2}^{2}+\frac{\beta}{3 \alpha}\|u\|_{6}^{6}\right) \\
&=-2 \operatorname{Re} \int_{-\infty}^{\infty} \int_{-\infty}^{\infty} \Delta \bar{u} u_{t} d x d y+\frac{2 \beta}{\alpha} \operatorname{Re} \int_{-\infty}^{\infty} \int_{-\infty}^{\infty}|u|^{4} \bar{u} u_{t} d x d y \\
&=-2 v\|\Delta u\|_{2}^{2}+2 \gamma\|\nabla u\|_{2}^{2}-\frac{2 \beta \kappa}{\alpha}\|u\|_{10}^{10}+\frac{2 \beta \gamma}{\alpha}\|u\|_{6}^{6} \\
&+ 2 \operatorname{Re}(\kappa+i \beta) \int_{-\infty}^{\infty} \int_{-\infty}^{\infty}|u|^{4} u \Delta \bar{u} d x d y \\
&+\frac{2 \beta}{\alpha} \operatorname{Re}(\nu+i \alpha) \int_{-\infty}^{\infty} \int_{-\infty}^{\infty}|u|^{4} \bar{u} \Delta u d x d y
\end{aligned}
$$

If $\int_{-\infty}^{\infty} \int_{-\infty}^{\infty}|u|^{4} u \Delta \bar{u} d x d y=A+i B$ then

$$
\begin{aligned}
2 \operatorname{Re}(\kappa+i \beta) & \int_{-\infty}^{\infty} \int_{-\infty}^{\infty}|u|^{4} u \Delta \bar{u} d x d y+\frac{2 \beta}{\alpha} \operatorname{Re}(v+i \alpha) \int_{-\infty}^{\infty} \int_{-\infty}^{\infty}|u|^{4} \bar{u} \Delta u d x d y \\
= & 2 \operatorname{Re}(\kappa+i \beta)(A+i B)+\frac{2 \beta}{\alpha} \operatorname{Re}(\nu+i \alpha)(A-i B) \\
= & 2\left(\kappa A-\beta B+\frac{2 \beta}{\alpha}(v A+\alpha B)\right. \\
= & \left(2 \kappa+\frac{2 \beta \nu}{\alpha}\right) \operatorname{Re} \int_{-\infty}^{\infty} \int_{-\infty}^{\infty}|u|^{4} u \Delta \bar{u} d x d y \\
= & -\left(2 \kappa+\frac{2 \beta \nu}{\alpha}\right) \int_{-\infty}^{\infty} \int_{-\infty}^{\infty}\left(3|u|^{4}\left|u_{x}\right|^{2}+2 \operatorname{Re} u^{3} \bar{u} u_{x}^{2}\right. \\
& \left.+3|u|^{4}\left|u_{y}\right|^{2}+2 \operatorname{Re} u^{3} \bar{u} u_{y}^{2}\right) d x d y \leq 0 .
\end{aligned}
$$

Combine (4.12) and (4.13), noting $\alpha, \beta, \nu>0, \kappa \geq 0$ and (2.11):

$$
\begin{aligned}
\partial_{t}\left(\|\nabla u\|_{2}^{2}+\frac{\beta}{2 \alpha}\|u\|_{6}^{6}\right) & \leq-2 v\|\Delta u\|_{2}^{2}+2 \gamma\|\nabla u\|_{2}^{2}-\frac{2 \beta \kappa}{\alpha}\|u\|_{10}^{10}+\frac{2 \beta \gamma}{\alpha}\|u\|_{6}^{6} \\
& \leq-2 c \nu\left\|\nabla^{2} u\right\|_{2}^{2}+2 \gamma\|\nabla u\|_{2}^{2}+\frac{2 \beta \gamma}{\alpha}\|u\|_{6}^{6} \\
& \leq-2 c v\left\|\nabla^{2} u\right\|_{2}^{2}+2 \gamma\|\nabla u\|_{2}^{2}+\frac{2 \beta|\gamma|}{\alpha} c_{6}^{6}\left\|\nabla^{2} u\right\|_{2}\|u\|_{2}^{4} \\
& \leq-2 c \nu\left\|\nabla^{2} u\right\|_{2}^{2}+2 \gamma\|\nabla u\|_{2}^{2}+2 c \nu\left\|\nabla^{2} u\right\|_{2}^{2}+\hat{c}\|u\|_{2}^{8} \\
& \leq 2 \gamma\|\nabla u\|_{2}^{2}+\hat{c} M_{0}^{8} .
\end{aligned}
$$


The last inequality is obtained via (4.2). Now (4.14) becomes

$$
\|\nabla u\|_{2}^{2}+\frac{\beta}{2 \alpha}\|u\|_{6}^{6} \leq\left\|\nabla u_{0}\right\|_{2}^{2}+\frac{\beta}{2 \alpha}\left\|u_{0}\right\|_{6}^{6}+\int_{0}^{t}\left(2 \gamma\|\nabla u\|_{2}^{2}+\hat{c} M_{0}^{8}\right) d \tau .
$$

Hence

$$
\|\nabla u\|_{2}^{2} \leq \tilde{c}+2 \gamma \int_{0}^{t}\|\nabla u\|_{2}^{2} d \tau+\hat{c} M_{0}^{6} T
$$

and by Gronwall's inequality $(0 \leq t \leq T)$

$$
\|\nabla u\|_{2}^{2} \leq\left(\tilde{c}+\hat{c} M_{0}^{8} T\right) \exp \{2|\gamma| t\} \leq M_{1}^{2} .
$$

This is exactly (4.7). Thus we have completed Step 1 and Step 2. Step 3 is similar (as shown in proof of Theorem 4.1).

It is indicated in [2] that for the 2D NLS (corresponding to $v=\kappa=0$ ), the solutions exist but blow up in finite time in the region $(\alpha \beta<0)$. While in the region $\alpha \beta>0$, the constant-amplitude solutions are modulationally stable. Whether the solution of the Cauchy problem for the 2D GL equation blows up in the whole region $\alpha \beta<0$ is unknown. When $D=1$, the GL equation in the super-critical case $(q=4)$ is a special version of the so-called generalized GL equation. Duan, Holmes and Titi have obtained the criterion for the global existence for the 1D generalized GL equation in a finite or infinite domain $[10,9]$. We would like to point out that if we replace our Cauchy problem on $R^{2}$ by the Cauchy problem with periodic boundary condition, the same results are still valid. The proof is quite similar and here we just list the following theorem.

THEOREM 4.4. For the $2 D$ complex $G L$ equation $(1.1)$ on $[0, L]^{2}$ with the initial condition $u_{0}(x, y) \in H^{3}\left([0, L]^{2}\right)$ and periodic boundary condition $u(x, y)=u(x+$ $L, y)=u(x, y+L)$, there exists a unique global solution $u \in C^{0}\left(H^{3}\left(R^{2}\right)\right) \cap$ $C^{1}\left(H^{1}\left(R^{2}\right)\right)$ if one of the following criteria holds: (1) $q=1 ;$ (2) $q=2$ and $|\beta| \leq \frac{\sqrt{5}}{2} \kappa ;(3) q=2$ and $\alpha \beta>0$.

We will investigate the 3D GL equation later and report our progress in the future.

\section{Acknowledgement}

The author thanks the referee and the associate editor for many helpful comments on an earlier version of this paper. This research was partially supported by a faculty research grant funded by the IBM. 


\section{References}

[1] M. J. Ablowitz and H. Segur, Solitons and the inverse scattering transform (North-Holland, 1981).

[2] M. Bartuccelli, P. Constantin, C. Doering, J. Gibbon and M. Gisselfalt, "On the possibility of soft and hard turbulence in the complex Ginzburg-Landau equation", Physica 44D (1990) 421-444.

[3] H. Brezis and T. Gallouet, "Nonlinear Schrödinger equations", Nonlinear Anal. 4 (1980) 677-681.

[4] C. Bu, "On well-posedness of the forced nonlinear Schrödinger equation", Applicable Anal. 46 (1992) 219-240.

[5] C. Bu, "The Ginzburg-Landau equation: Posed in a quarter plane", J. Math. Anal. Appl. 176(1993) 493-520.

[6] R. Carroll, Abstract methods in partial differential equations (Harper and Row, New York, 1969).

[7] R. Carroll, "On the forced nonlinear Schrödinger equation", Japan J. Appl. Math. 7 (1990) 321344.

[8] R. Carroll and C. Bu, "Solutions of the forced nonlinear Schrödinger equation (NLS) using PDE techniques", Applicable Anal. 41 (1991) 33-51.

[9] J. Duan and P. Holmes, "On the Cauchy problem for a generalized Ginzburg-Landau equation", preprint, 1992.

[10] J. Duan, P. Holmes and E. Titi, "Regularity approximation and asymptotic dynamics for a generalized Ginzburg-Landau equation", Nonlinearity 5 (1992) 1303-1314.

[11] A. Fokas, "An initial-boundary value problem for the nonlinear Schrödinger equation", Physica 35D (1989) 167-185.

[12] J. Ghidaglia and R. Heron, "Dimensions of attractors associated to the Ginzburg-Landau equation", Physica 28D (1987) 282-304.

[13] L. Hocking, K. Stewartson and J. Stuart, "A nonlinear instability burst in plane parallel flow", $J$. Fluid Mech. 51 (1972).

[14] D. Kaup, Wave Phenomena (North-Holland, 1984).

[15] C. Lange and A. Newell, "A stability crireion for the envelope equations", SIAM J. Appl. Math. 27 (1974) $441-456$.

[16] J. Lions, Quelques méthodes de résolution des problémes aux limites non linéaires (Dunod, Paris, 1969).

[17] A. Newell and J. Whitehead, "Finite bandwidth, finite amplitude convection", J. Fluid Mech. 38 (1969).

[18] P. Newton, "Wave interactions in the singular Zakhorov systems", J. Math. Phys. 29 (1988) 2245-2249.

[19] L. Nirenberg, On elliptic partial differential equations (Pisa, 1959) 115-162.

[20] A. Pazy, Semigroups of linear operators and applications to PDE (Springer, New York, 1983).

[21] I. Segal, "Nonlinear semigroups", Ann. Math. 78 (1963) 339-364.

[22] K. Stewartson and J. Stuart, "A non-linear instability theory for a wave system in plane Poiseville flow", J. Fluid Mech. 48 (1971).

[23] W. Strauss, "Nonlinear scattering theory at low energy", J. Funct. Anal. 41 (1981) 110-132.

[24] W. Strauss, Nonlinear wave equations (CBMS, American Mathematical Society, Rhode Island, 1989).

[25] M. Tsutsumi, "On smooth solutions to the initial-boundary value problem for the nonlinear Schrödinger equation", Nonlinear Anal. 13 (1989) 1051-1056.

[26] Y. Tsutsumi, "Global solutions to the nonlinear Schrödinger equation", Comm. Partial Differential Equations 8 (1983) 1337-1374. 